\title{
In Fondo ALLA SCRITTURA (LA Scrittura di Chi Sta in Fondo) Le Testimonianze Scritte Della Gente Comune
}

Fabio Caffarena

ABSTRACT La storia, soprattutto quella contemporanea, può essere ripercorsa attingendo alle consuete fonti istituzionali, legate ai classici paradigmi interpretativi economici e istituzionali, ma anche privilegiando gli aspetti sociali, utilizzando l'inesauribile miniera di testimonianze scritte ed orali prodotte dalla gente comune. Tali fonti consentono uno sguardo "dal basso" degli eventi, ma tale prospettiva, per nulla trascurabile e dotata di una sempre più solida concettualizzazione metodologica, è ancora alla ricerca di una completa legittimazione storiografica.

PAROLE CHIAVE macro-storia; micro-storia; archivi; gente comune; soggettività; scritture popolari. 
RESUMO A história, especialmente a contemporânea, pode ser abordada utilizandose as costumeiras fontes institucionais, ligadas aos clássicos paradigmas econômicos e institucionais, mas também privilegiando os aspectos sociais, utilizando a mina inesgotável de testemunhos escritos e orais produzidos pela gente comum. Essas fontes permitem que se olhe "de baixo" para os eventos, mas essa perspectiva, que não pode ser ignorada e adquire uma conceituação metodológica cada vez mais consistente, está ainda buscando uma legitimação historiográfica completa.

PAL NTRAS-CHAVE macro-história; micro-história; arquivos; gentecomum; subjetividade; escritos populares.

ABSTRACT History, especially contemporary history, can be written by drawing on the usual institutional sources, and deploying the classical interpretative paradigms focussed on institutions and the economy. But history can also be written with an emphasis on its social dimensions, by delving into the inexhaustible mine of testimonies left by ordinary people. Such sources allow events to be viewed "from below" Yet despite the importance of this second approach, and despite the increasing solidity ofits methodological foundations, it has still to attain complete historiographical legitimacy.

KEYWORDS macro-history; micro-history; archives; ordinary people; subjectivity; popular writings. 


\section{Fondi e fonti*}

[...]Lastorianon esiste [...]. Vostro nonno ha scritto la sua storia? E vostro padre? E il mio? E i nostri avoli e trisavoli?... Sono discesi a marcire nella terra né più e né meno che come foglie, senza lasciare storia... [...] E il gorgoglio delle loro viscere vuote? E la voce della loro fame? Credete che si sentirà, nella storia? Che ci sarà uno storico che avrà orecchio talmente fino da sentirlo? [...].

Leonardo Sciascia, Il Consiglio d'Egitto, 1963.

Uomini senza archivi e senza volto: cosi gli storici moderni - afferma Aron Ja. Gurevic - hanno definito il popolo in un'epoca in cui gli era precluso un accesso diretto agli strumenti con

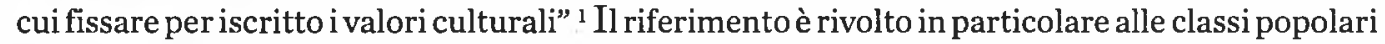
del periodo medievale, tuttavia il rapporto privilegiato ed escludente fra il "diritto alla storia" e la scrittura, come forma di conservazione di eventi e memoria, costituisce una dinamica di lungo periodo che arriva fino all'epoca contemporanea. Uomini senza tracce di carta depositate nei raccoglitori istituzionali della memoria, ${ }^{3}$ quindi senza fondi, nell'accezione archivistica dei significati più congeniale allo storico. Questo almeno in apparenza.

L'assonanza casuale crea un concatenamento di significati tra fondi e fonti, sebbene i termini si possano collocare agli estremi: la fonte è un punto di partenza, il fondo ciò che resta, ciò che è filtrato dalle maglie del tempo. Ma per lo storico - coincidenza degli opposti - si tratta di un concatenamento virtuoso ed efficace poiché ciò che resta, come fondo di un magazzino di eventie relazioni, costituisce il punto di partenza dell'indagine sul passato più o meno recente. Da questa prospettiva, guardare dal fondo significa innanzitutto guardare idocumenti, ifondi d'archivio: ogni traccia sul terreno può essere un documento, ogni terreno un archivio. La forma che prendono il tempo e gli eventi è plasmata dal punto di vista assunto e dalle relative tracce - le fonti - che è possibile trovare: è quindi una forma mutevole.

\footnotetext{
* Questo testo raccoglie, ampliandole, alcune riflessioni presentate al Convegno Guardare dal fondo - Le forme del tempo, a cura del Centro culturale Candiani di Mestre (Venezia), 14 aprile 2005.

1. Cfr. A. Ja. Gurevic, Contadini e santi. Problemi della cultura popolare nel Medioevo, Torino, Einaudi, $2000, \mathrm{p}$. VIII.

2. Ibidem, p. IX.

3. Sulla definizione di fondo in ambito archivistico, inteso come "complesso documentario che abbia un carattere di unitarietà", cfr. P. Carucci, Le fonti archivistiche: ordinamento e conservazione, Roma, N.I.S, 1993, p.201.
} 
La storia della contemporaneità propone nuove problematiche metodologiche costituite dal fatto che le fonti a disposizione sono talmente numerose e tipologicamente differenziate da non poter essere contenute tutte negli archivi, almeno nelle forme consuete in cui comunemente si intendono questi strati sovrapposti e organizzati del passato: basta frequentare l'archivio di un qualsiasi Ente istituzionale attivo per rendersi immediatamente conto della proliferazione incontrollabile di carta che aumenta in modo esponenziale nel corso degli anni. Spesso la documentazione più ordinata e fruibile risulta essere proprio quella più antica, mentre la più recente, in barba agli snellimenti per ora solo teorici ed illusori dell'era informatica, è talvolta semplicemente accatastata in depositi di fortuna. Ad oggi sono gli archivi, fisicamente, a non contenere più la carta ed in un certo senso si potrebbe dire che l'epoca contemporanea porta con sè un processo quasi fisiologico di disordine - quando non di dispersione - delle fonti: fenomeno che si potrebbe interpretare quasi come un sintomo visibile dell'estrema complessità di quest'epoca. I fondi diventano così inesplorabili baratri d'archivio, in attesa di smaterializzarsi, di migrare nel Web trasformandosi in non meno inquietanti database elettronici, in agglomerati di bytes. ${ }^{4}$

La moderna - e per certi aspetti paradossale - propensione alla dispersione di informazioni non è solo una questione archivistica, ma coinvolge anche un elemento fondamentale per la ricostruzione storica dell'epoca contemporanea di cui lo storico dell'antichità deve necessariamente fare a meno: la memoria, la testimonianza diretta, il fondo di magazzino di esperienze vissute il cui utilizzo in ambito storiografico è oggetto di un ampio dibattito. ${ }^{5}$

\section{Uno sguardo sottosopra...}

Ad oggi il rapporto fra ricostruzione storica e documenti presuppone il primato delle carte ufficiali e dei contenitori archivistici che raccolgono documentazione burocratica, espressione degli enti e delle istituzioni. ${ }^{6}$ Meno rappresentati sono gli individui, i gruppi sociali, a meno che la loro vita ed il loro operato non siano strettamente connessi ad una dimensione pubblica: la gente comune insomma, per quanto questa definizione sia un'etichetta omologante imposta ad individui molto eterogenei per cultura ed esperienze.

La storia non può essere ridotta ad una collezione di fatti e occorre ridare volume agli eventi liberandoli dagli schemi ideali, dalle scuole di pensiero storiografico, che restituiscono in alcuni casi una narrazione storica affidata solo alle grandi categorie concettuali, ai quadri indifferenziati e amorfi privi delle sfaccettature e della complessità del presente: "la storia - ha rilevato efficacemente Franco Ferrarotti - non è un fiume, relativamente unilineare, e neppure un torrente. $\grave{E}$ piuttosto un delta a più rami con uno sbocco dai contorni incerti, coperto da

4. Cfr. S. Vitali, Passato digitale. Le fonti dello storico nell'era del computer, Milano, Mondadori, 2004.

5. Sui rapporti fra storia e memoria e sul ruolo della finzione nella ricostruzione storica cfr. K. Pomian, Che cosè la storia, Milano, Mondadori, 2001. Sull'utilizzo della soggettività in ambito storiografico cfr. G. De Luna, La passione e la ragione. Fonti e metodi dello storico contemporaneo, Milano, La Nuova Italia, 2001; Q. Antonelli, A. Iuso (a cura di), Vite di carta, Napoli, Ĺancora del Mediterraneo, 2000 ed il contributo di A. Gibelli, Soggettività e storia del Novecento, in «Ventesimo secolo», 4 (1992).

6. Cfr. I. Zanni Rosiello, Archivi e memoria storica, Bologna, Il Mulino, 1987. 
cimmeriche nebbie tanto che a nessuno è dato di descrivere con accuratezza il complesso divaricarsi. Si comprende l'ansia semplificatrice degli storici tradizionali, di quelli che preferiscono battere i sentieri relativamente sgombri e rettilinei dell'histoire-bataille invece dei tortuosi cammini dell'histoire-homme. La loro cura filologica merita ogni apprezzamento, ma la loro prospettiva va ampliata in maniera piuttosto sostanziale. Ai «documenti» e ai «monumenti» sono da aggiungersi le testimonianze [...] La storia così «complessificata» sfugge alle categorie tradizionalizzate, forse comode ma anguste [...] Da storia storica, più o meno marmorizzata, si scioglie nella fluidità problematica delle storie di vita. La storia dei principi deve accogliere, e accettare di venire riscritta, come storia dei sudditi. Storico e vissuto cominciano a dar luogo a una sottile, inesplorata dialettica relazionale"7

Questo salto storiografico implica una scelta difondo - nel senso di fondamento prospettico - e di fonti: un primato del vissuto sulla cronaca evenemenziale. Come ha affermato Michel Vovelle in un saggio dedicato a La nuova storia, ogni epoca crea - e lascia - le fonti utili a ripercorrerla ${ }^{8}$ e l'epoca contemporanea mette a disposizione della ricerca fonti qualitative: penso alle testimonianze, alle parole labili affidate alla volatilità delle voci, ma anche alle innumerevoli lettere, diari e memorie autobiografiche scritti dalla gente comune. Veri e propri fondi a cielo aperto cui attingere:

"Si immagini ora un uomo a cui, - ha scritto Primo Levi - insieme con le persone amate, vengano tolti la sua casa, le sue abitudini, i suoi abiti, tutto infine, letteralmente tutto quanto possiede: sarà un uomo vuoto, ridotto a sofferenza e bisogno, dimentico di dignità e discernimento, poiché accade facilmente, a chi ha perso tutto, di perdere se stesso; tale quindi, che si potrà a cuor leggero decidere della sua vita o morte al di fuori di ogni senso di affinità umana; nel caso più fortunato, in base ad un puro giudizio di utilità. Si comprenderà allora il duplice significato del termine «Campo di annientamento», e sarà chiaro che cosa intendo esprimere con questa frase: giacere sul fondo.

[...] Eccomi dunque sul fondo. A dare un colpo di spugna al passato e al futuro si impara assai presto, se il bisogno preme. ${ }^{?}$

Per immaginare questo uomo privato del proprio vissuto, per sondare il tremendo fondo in cui è precipitato e dove si è spento ogni senso di pietà umana, le testimonianze dirette (orali e scritte) rappresentano evidentemente gli strumenti più efficaci, in grado di dare maggiore profondità e spessore alla ricostruzione storica: una tridimensionalità impossibile utilizzando esclusivamente i dati quantitativi dell'Olocausto, per quanto impressionanti e significativi.

In questo senso anche un approccio storiografico classico, che presuppone il primato delle fonti scritte, dovrebbe accettare ormai l'ingombrante eredità cartacea, se non quella sonora, delle classi popolari. Una dote che fornisce allo storico raffinati strumenti d'indagine che consente di rivalutare

7. Cfr. F. Ferrarotti, La storia e il quotidiano, Roma-Bari, Laterza, 1986, pp. 116-117.

8. Cfr. M. Vovelle, La nuova storia, Milano, Mondadori, 1980.

9. P. Levi, Se questo è un uomo. La tregua, Torino, Einaudi, 2003, pp. 23-31. 
il ruolo assunto da individui subalterni, talvolta emarginati e perseguitati, che comunque sono stati protagonisti del XX secolo e non certo comparse anonime dal ruolo secondario. ${ }^{10}$

La gente comune ha lasciato - e continua a lasciare - archivi di voci, quando queste sono state raccolte, trascritte e impresse su un supporto magnetico: ${ }^{11}$ la testimonianza orale tuttavia non si fonda qualitativamente solo sulla percezione uditiva, ma anche su una miriade di suggestioni fornite dall'espressività gestuale del narratore, infine dall'interazione con l'interlocutore a cui si racconta la propria storia di vita. La fonte scritta risulta mutila di questo impianto espressivo, che costituisce un valore aggiunto alla testimonianza.

Le testimonianze scritte della gente comune danno vita invece ad archivi involontari, per quanto sfuggenti e anarchici, senza scaffali ed inventari. Sono carte che tendenzialmente rifuggono alla conservazione sistematica: occorre scovarle nei fondi delle cantine, nei cassetti. Si tratta quasi sempre di sedimentazioni spontanee di scritti depositati negli anfratti delle case, finite ai margini fisici della quotidianità familiare e del ricordo.$^{12}$ La loro conservazione è legata spesso all'oblio in cui sono precipitati o, al contrario, al rapporto affettivo che ne impedisce la distruzione da parte dei possessori, legati da rapporti di parentela o amicizia con gli scriventi. Questi testi lasciati dalla gente comune, come tracciati di un sismografo, costituiscono l'indicatore di un'imponente spinta chirografica avvenuta non appena le masse popolari hanno dovuto impossessarsi, per quanto precariamente, di una nuova tecnologia comunicativa: la scrittura. L'uso di questa tecnica da parte della gente comune è attestato già nel periodo medievale e moderno, ma si diffonde soprattutto tra il XIX e il XX secolo. Non si tratta di un fenomeno legato esclusivamente al consolidamento dei percorsi scolatici e al conseguente maggior tasso di alfabetismo, ma di una pratica indotta soprattutto dal bisogno di comunicare a distanza, di fissare la consueta comunicazione orale su un supporto in grado di abbattere le distanze e ricucire almeno idealmente gli strappi sociali e culturali causati dagli eventi separatori della modernità. ${ }^{13}$

La Grande emigrazione $\mathrm{e}^{14} \mathrm{e}$ la Prima guerra mondiale ${ }^{15}$ costituiscono a questo proposito eventi chiave che smuovono uno strato sepolto - fragile ma fertile - di pratiche scrittorie, trasformandole

10. Nel saggio dedicato alla Gente non comune (Milano, Rizzoli, 2000), E. J. Hobsbawm ha scritto che "quello che [le persone comuni] hanno pensato e fatto è tutt'altro che trascurabile: era in grado di influire, e ha influito, sulla cultura e sugli avvenimenti, e questo non è mai stato cosi vero come nel XX secolo" (p.8).

11. Si pensi ad esempio agli imprescindibili lavori di N. Revelli. Per una panoramica dei centri internazionali preposti al recupero ed allo studio delle fonti oral i cfr. C. Bermani (a cura di), Introduzione alla storia orale. Esperienze di ricerca, Roma, Odradek, 2001. Vol. II (Indirizzi e siti internet della Storia orale, a cura della Società di Mutuo soccorso "Ernesto de Martino" di Venezia). Sulle problematiche connesse all'utilizzo di queste fonti cfr. G. Contini; A. Martini, Verba manent. L'uso delle fonti orali per la storia contemporanea, Roma, N.I.S., 1993.

12. A questo scopo in Italia sono sorti centri di raccolta delle testimonianze scritte della gente comune: tra i principali l'Archivio della Scrittura Popolare di Trento (A.S.P.), l'Archivio Ligure della Scrittura Popolare di Genova (A.L.S.P.) e la Fondazione Archivio Diaristico Nazionale di Pieve Santo Stefano (A.D.N.).

13. Su questi aspetti cfr. P. Conti; G. Franchini; A. Gibelli (a cura di), Storie di gente comune, Acqui Terme, Editrice Impressioni Grafiche, 2002.

14. Sui nuovi paradigmi interpretativi dei fenomeni migratori cfr. M. Tirabassi (a cura di), Itinera. Paradigmi delle migrazioni italiane, Torino, Fondazione Giovanni Agnelli, 2005. Sulle testimonianze degli emigranti cfr. E. Franzina, Merica! Merica. Emigrazione e colonizzazione nelle lettere dei contadini veneti e friulani in America Latina (1876-1902), Milano, Feltrinelli, 1979 (Verona, Cierre Edizioni, 1994²); A. Gibelli; F. Caffarena, Le lettere degli emigranti, in P. Bevilacqua; A. De Clementi; E. Franzina (a cura di), Storia dell'emigrazione italiana-Partenze, Roma, Donzelli, 2001 e il lavoro di F. Croci; G. Bonfiglio, El baúl de la memoria. Testimonios escritos de inmigrantes italianos en el Perú, Lima, Fondo Editorial del Congreso de la Republica del Perú, 2002, che utilizza testimonianze provenienti da archivi familiari ritrovati in Perù. In particolare, sulle lettere di chiamata, che rappresentano una tipologia di testi ancora poco studiata, cfr. l'articolato studio di F. Croci, La parta per le Americhe. Migrazioni italiane a São Paulo: lettere di chiamata e ricongiungimentifamiliari, Università di Genova, Tesi di dottorato XVIII ciclo in Scienze Storico Filosofiche (indirizzo Storia contemporanea) discussa nell'aprile 2007.

15. Per quanto riguarda il vissuto bellico dei combattenti durante la Grande Guerra, oltre al classico studio di A. Gibelli, L'officina della guerra. La Grande Guerra e le trasformazioni del mondo mentale, Torino, Bollati-Boringhieri $2007^{3}$ (con una nuova ed ampia postfazione che riassume 
in efficaci strumenti comunicativi. Ł̇ ipotizzabile infatti che questi eventi portino in superficie usi sotterranei della scrittura talvolta già attivati come supporto della memoria, senza una funzione comunicativa, come ad esempio la piccola contabilità domestica. Ma quando dall'annotazione contabile si passa al racconto esperienziale, scrivere significa riaffermare la soggettività, lasciando tracce autografe del proprio passaggio.

Ogni documento ed ogni racconto diventano strumenti utili per sondare la dimensione psicologica e sociale degli individui, rendendo possibile la ricostruzione di una storia dei comportamenti e delle relazioni. ${ }^{16}$ Una storia che prende forma anche attraverso le contaminazioni linguistiche che si possono ritrovare nelle missive degli emigranti, come segno di commistione culturale fra due sponde dell'oceano e sintomo di progressivo radicamento nella cultura del paese di arrivo.

Le testimonianze della gente comune costituiscono un punto di osservazione interno agli eventi e sono in grado di fornire all'osservatore scorci di storia e di storie non trascurabili. È così possibile cogliere le ricadute della Grande storia sulla quotidianità soggettiva di uomini, donne e bambini, evidenziando le complesse connessioni che le esperienze personali stabiliscono con il piano generale della storia. Ogni testo è evidentemente una risposta soggettiva agli eventi in corso, ma quando tante storie di vita possono essere messe in relazione, confrontate secondo un'accurata griglia metodologico-interpretativa, si riesce a delineare traiettorie piuttosto definite, seppur nel loro moto irregolare: per l'emigrazione si dovrà ad esempio sfumare l'esclusiva dinamica pauperistica legata a fattori espulsivi (dalla terra di origine) ed attrattivi (dei paesi di adozione), introducendo svariate motivazioni personali, familiari e comunitarie alla base della mobilità di tanti individui. E sarà possibile scomporre gli eserciti in soldati, in individui collettivi (i piccoli gruppi di combattenti che configurano un surrogato di famiglia), fino agli individui singoli, sondandone le intime paure, i contrasti latenti o laceranti tra consenso e rifiuto. "Se pure non offrono una testimonianza fedele degli eventi, - ha rilevato a questo proposito Stefano Catucci - le lettere, i diari e le memorie autobiografiche della Grande Guerra sono comunque la traccia più significativa dell' «atto configurante» con il quale i soldati semplici e gli ufficiali, i contadini, i borghesi e gli intellettuali hanno cercato, a diversi livelli e su vasta scala, di dar forma all'esperienza di se stessi nel nuovo mondo in cui si trovavano scaraventati" ${ }^{\prime 7}$

Occorre sottolineare come le tante memorie soggettive non sempre coincidano con la memoria collettiva, tantomeno con la fattualità storica: ma è proprio in questa frattura fra storia e memoria, intesa come rielaborazione singola del vissuto, che si possono trovare risposte utili per capire i processi di adattamento e di resistenza agli eventi. Una frattura che si produce lungo il confine mutevole che scorre fra accadimenti e ricordo, creando storia: insomma, le rappresentazioni della realta fornite dalle fonti soggettive sono altrettanto importanti della ricostruzione oggettiva degli eventi e, come sostiene Krzysztof Pomian, "senza la consapevolezza dell'esistenza di un confine tra il regno della realtà e quello in cui è la finzione a esercitare un pieno potere non è possibile avere alcuna storia" 18

il percorso storiografico degli ultimi venti anni), segnalo il mio studio, Lettere dalla Grande Guerra: il caso italiano. Scritture del quotidiano, monumenti della memoria, fonti per la storia, Milano, Unicopli, 2005, che rinvia ad un cospicuo apparato bibliografico e di fonti.

16. Cfr. P. Jedlowski, Storie comuni. La narrazione nella vita quotidiana, Milano, Mondadori, 2000 e P. Sorcinelli, Il quotidiano e i sentimenti. Viaggio nella storia sociale, Milano, Mondadori, 2002, in cui l'autore, intersecando proficuamente prospettive sociologiche e storiche, ha ripercorso le tracce di storia che restano nella quotidianità fornendo una rassegna delle possibili fonti in cui si trovano depositate tante minime, ma utilissime, storie di vita. Cfr. anche D. Calanca, Legami. Relazioni familiari nel Novecento, Bologna, Bononia University Press, 2005. 
La memoria scompone e ricompone continuamente il ricordo, lo rimodella in base alle sollecitazioni indotte dal succedersi delle esperienze e talvolta da convenienze più o meno volontarie: in questo senso, ciò che un testimone e una testimonianza tacciono è al trettanto importante di ciò che rivelano. Le innumerevoli rappresentazioni mentali e le sfasature della memoria, intese come percezioni della [propria] storia, acquistano sempre maggior rilievo quanto più vengono miniaturizzate, declinate al soggettivo. Indubbiamente lavorare con le fonti individuali implica attingere a strumenti cognitivi non sempre posseduti dallo storico, a categorie socio-antropologiche che conducono ad un proficuo percorso interdisciplinare.

\section{Testi e con-testi}

Antonio Gramsci il 16 novembre 1931 scrive dal carcere una lettera alla sorella Teresina particolarmente efficace per accedere al paesaggio mentale popolare ed ai suoi abitanti:

[...] molti tipi di villaggio [...] per fare sfoggio di cultura, raccattavano dai romanzi popolari delle grandi frasi e poi le facevano entrare a dritta e a traversa nella conversazione per far stupire i contadini. Allo stesso modo le beghine ripetono il latino delle preghiere contenute nella Filotea: ti ricordi che zia Grazia credeva fosse esistita una «donna Bisodia» molto pia, tanto che il suo nome veniva sempre ripetuto nel Pater noster? Era il «dona nobis hodie» che lei, come molte altre, leggeva «donna Bisodia» e impersonava in una donna del tempo passato, quando tutti andavano in Chiesa e c'era ancora un po' di religione in questo mondo. - Si potrebbe scrivere una novella su questa «donna Bisodia» immaginaria che era portata a modello: quante volte zia Grazia avrà detto a Grazietta, a Emma e anche a te forse: «Ah, tu non sei certo come donna Bisodia!» quando non volevate andare a confessarvi per l'obbligo pasquale. Adesso tu potrai raccontare ai tuoi bambini questa storia [... $]^{19}$

In una prospettiva liminare fra antropologia e storia, ciò che interessa non è l'esistenza storicizzabile di "donna Bisodia", ma la sua effettiva presenza nell'immaginario collettivo, che poi si traduce in una presenza quanto mai reale e storicamente rilevabile del vissuto: come l'arrivo di Lenin nelle piazze di alcuni sperduti paesini italiani (quasi sempre riconducibile all'effettiva presenza di militanti comunisti o sindacalisti locali). Esempi, aneddoti, che si potrebbero moltiplicare, mettendo in rilievo la necessità di una continua e puntuale contestualizzazione storica, di incrociare le fonti, per ridimensionare e in qualche caso confutare la "fantasia mitologizzante" ${ }^{20}$ Ciò non implica tuttavia

19. Sulla diffusione nell'immaginario popolare di «donna Bisodia» - come santa o donna malvagia - cfr. E. Sanguineti, Giornalino 1973-1975, Torino, Einaudi, 1976, pp. 185-187.

20. F. Ferrarotti, cit., p.184. 
l'attribuzione di una patente di maggior attendibilità di una fonte rispetto ad un'altra. La dialettica e la non sovrapponibilità tra le varie tracce del passato non fa altro che evidenziare allo storico la faglia instabile tra storia e memoria. Una precarietà che a ben vedere è anche virtuosa.

\section{...uno sguardo invadente?}

L'inserimentodelle fonti popolari nella panoplia di strumenti che armano lo storico contemporaneo non è stato affatto - e non è tuttora - indolore: per alcuni si tratta di uno sguardo storiografico che tenderebbe a prevaricare le consolidate metodologie di ricerca, provocando un "eccesso di memoria":21 "a forza di parlare di sé, - sostiene Alberto Cavaglion - la memoria è degenerata nel culto della memoria, che è altra cosa; oppure facendo leva sui ritardi della storiografia, ha cercato di sostituirsi alla storia, anche qui facendo un cattivo uso di se stessa e generando non pochi malintesi [...] la storia, o sarebbe meglio dire la ricerca storiografica, in odio alla storiografia politicodiplomatica della generazione precedente, ha coltivato in maniera altrettanto sproporzionata, ed eccessiva, il sogno di un'inverosimile "ego-storia", insomma di una storia in cui la soggettività - strumento classico della memoria - ha finito con il prevalere sulla ricerca stessa e sul dominio delle fonti archivistiche e scritte" ${ }^{22}$

Annette Becker e Stéphane Audoin-Rouzeau, riferendosi alle testimonianze dei combattenti francesi della Grande Guerra, sostengono similmente che "le cose si sono nondimeno complicate in conseguenza del fatto che i veterani non si sono limitati a rivendicare il proprio statuto - certo incontestabile - di testimoni dell'esperienza di guerra, ma hanno voluto assumere anche quello di storici, e di storici determinati ad assicurarsi l'esclusività del discorso su quel vissuto" 23

Queste considerazioni, che evocano "una certa forma di dittatura"24 delle testimonianze dirette, possono essere estese a tutti i campi di applicazione della storia vista dal fondo, inteso questa volta come punto più basso. L'utilizzo delle fonti popolari non si è trasformato ancora in un orizzonte storiografico condiviso in quanto far interagire biografie minime di persone comuni e quadri d'assieme è tutt'altro che una sfida agevole. La storia contemporanea tuttavia non può rinunciare ad occuparsi della soggettività, del suo fondo e delle sue fonti.

Addentrarsi nella "ego-storia", con le dovute cautele, non significa affatto sprofondare in una sorta di voyeurismo del passato e delle sue storie. Significa invece acquisire indizi più definiti per comprendere la storia caotica della modernità, sfruttare gli "atti configuranti” dei singoli per ricomporre il quadro assolutamente disarticolato degli eventi e della loro memoria.

21. In generale, su questo aspetto, cfr. C. Maier, Un eccesso dimemoria? Riflessioni sulla storia, la malinconia e la negazione, in «Parole chiave»,9 (1995). Cfr. anche A. Tarpino, Memoria e crisi della società del ricordo, in "Memoria e Ricerca», 10 (2002).

22. Cfr. A. Cavaglion, Sui vuoti di memoria, in Q. Antonelli, A. Iuso (a cura di), Vite di carta... cit., pp. 218-222.

23. Cfr. S. Audoin-Rouzeau; A.Becker, La violenza, la crociata, il lutto. La Grande Guerra e la storia del Novecento, Torino, Einaudi, 2002 , p. 29.

24. Cfr. S. Audoin-Rouzeau; - A.Becker, cit., p. 30. 


\section{Bibliografia}

Q. Antonelli, A. Iuso (a cura di), Vite di carta, Napoli, L'ancora del Mediterraneo, 2000.

S. Audoin-Rouzeau; A.Becker, La violenza, la crociata, il lutto. La Grande Guerra e la storia del Novecento, Torino, Einaudi, 2002.

C. Bermani (a cura di), Introduzione alla storia orale. Esperienze di ricerca, Roma, Odradek, 2001. Vol. II

F. Caffarena, Lettere dalla Grande Guerra: il caso italiano. Scritture del quotidiano, monumenti della memoria, fonti per la storia, Milano, Unicopli, 2005.

D. Calanca, Legami. Relazioni familiari nel Novecento, Bologna, Bononia University Press, 2005.

P. Carucci, Le fonti archivistiche: ordinamento e conservazione, Roma, N.I.S, 1993, p.201.

S. Catucci, Per una filosofia povera. La Grande Guerra, l'esperienza, il senso: a partire da Lukács, Torino, Bollati Boringhieri, 2003.

G. Contini; A. Martini, Verba manent. L'uso delle fonti oraliper la storia contemporanea, Roma, N.I.S., 1993.

P. Conti; G. Franchini; A. Gibelli (a cura di), Storie di gente comune, Acqui Terme, Editrice Impressioni Grafiche, 2002.

F. Croci;- G. Bonfiglio, El baúl de la memoria. Testimonios escritos de inmigrantes italianos en el Perú, Lima, Fondo Editorial del Congreso de la Republica del Perú, 2002.

F. Croci, La porta per le Americhe. Migrazioni italiane a São Paulo: lettere di chiamata e ricongiungimenti familiari, Università di Genova, Tesi di dottorato XVIII ciclo in Scienze Storico Filosofiche (indirizzo Storia contemporanea) discussa nell'aprile 2007.

G. De Luna, La passione e la ragione. Fonti e metodi dello storico contemporaneo, Milano, La Nuova Italia, 2001.

F. Ferrarotti, La storia e il quotidiano, Roma-Bari, Laterza, 1986.

E. Franzina, Merica! Merica. Emigrazione e colonizzazione nelle lettere dei contadini veneti e friulani in America Latina (1876-1902), Milano, Feltrinelli, 1979 (Verona, Cierre Edizioni, 1994²).

A. Gibelli; F. Caffarena, Le lettere degli emigranti, in P. Bevilacqua; A. De Clementi; E. Franzina (a cura di), Storia dell'emigrazione italiana. Partenze, Roma, Donzelli, 2001.

A. Gibelli, L'officina della guerra. La Grande Guerra e le trasformazioni del mondo mentale, Torino, Bollati-Boringhieri, $2007^{3}$.

A. Gibelli, Soggettività e storia del Novecento, in «Ventesimo secolo», 4 (1992).

A. Ja. Gurevic, Contadini e santi. Problemi della cultura popolare nel Medioevo, Torino, Einaudi, 2000.

P. Jedlowski, Storie comuni. La narrazione nella vita quotidiana, Milano, Mondadori, 2000.

P. Levi, Se questo è un uomo. La tregua, Torino, Einaudi, 2003.

C. Maier, Un eccesso di memoria? Riflessioni sulla storia, la malinconia e la negazione, in «Parole chiave», 9 (1995).

K. Pomian, Che cosè la storia, Milano, Mondadori, 2001.

E. Sanguineti, Giornalino 1973-1975, Torino, Einaudi, 1976, pp. 185-187.

P. Sorcinelli, Il quotidiano e i sentimenti. Viaggio nella storia sociale, Milano, Mondadori, 2002.

A. Tarpino, Memoria e crisi della società del ricordo, in «Memoria e Ricerca», 10 (2002).

M. Tirabassi (a cura di), Itinera. Paradigmi delle migrazioni italiane, Torino, Fondazione Giovanni Agnelli, 2005.

S. Vitali, Passato digitale. Le fonti dello storico nell'era del computer, Milano, Mondadori, 2004.

M. Vovelle, La nuova storia, Milano, Mondadori, 1980.

I. Zanni Rosiello, Archivi e memoria storica, Bologna, Il Mulino, 1987. 